\title{
On the computation of $\lambda$-contractive sets for linear constrained systems
}

\author{
Moritz Schulze Darup ${ }^{\dagger}$ and Mark Cannon ${ }^{\dagger}$
}

\begin{abstract}
We present two theoretical results on the computation of $\lambda$-contractive sets for linear systems with state and input constraints. First, we show that it is possible to a priori compute a number of iterations that is sufficient to approximate the maximal $\lambda$-contractive set with a given precision using 1-step sets. Second, based on the former result, we provide a procedure for choosing $\lambda$ so that the associated maximal $\lambda$ contractive set is guaranteed to approximate the maximal controlled invariant set with a given accuracy.
\end{abstract}

Keywords. Linear systems, constrained control, $\lambda$-contractive sets, geometric methods.

Preamble. This is a preprint of an article to appear in IEEE Transactions on Automatic control.

\section{Introduction and Problem Statement}

The concept of $\lambda$-contraction is widely used in control theory (see 1 for an excellent overview). For linear discrete-time systems

$$
x(k+1)=A x(k)+B u(k)
$$

with state and input constraints of the form

$$
x(k) \in \mathcal{X} \text { and } u(k) \in \mathcal{U} \text { for every } k \in \mathbb{N},
$$

the construction, the application and many fundamental properties of $\lambda$-contractive sets are well-known (see, e.g., [1, 2]). The computation of $\lambda$-contractive sets often builds on the repeated evaluation of the mapping

$$
\mathcal{Q}_{1}^{\lambda}(\mathcal{D}):=\{x \in \mathcal{X} \mid \exists u \in \mathcal{U}: A x+B u \in \lambda \mathcal{D}\}
$$

for a given set $\mathcal{D} \subset \mathbb{R}^{n}$ and a fixed $\lambda \in(0,1]$. This leads to a sequence of sets defined according to $\mathcal{Q}_{0}^{\lambda}(\mathcal{D})=\mathcal{D}$ and

$$
\mathcal{Q}_{k+1}^{\lambda}(\mathcal{D}):=\mathcal{Q}_{1}^{\lambda}\left(\mathcal{Q}_{k}^{\lambda}(\mathcal{D})\right)
$$

for $k \in \mathbb{N}$. Sequences of the form (4) were first addressed for the special case $\lambda=1$ (see [3, 4, 5, 6]). Later, the case of $\lambda \in(0,1)$, which is more relevant here, was considered (see,

${ }^{\dagger}$ M. Schulze Darup and M. Cannon are with the Control Group, Department of Engineering Science, University of Oxford, Parks Road, Oxford OX1 3PJ, UK. E-mail: moritz.schulzedarup@rub.de. 
e.g., 2]). For the special choice $\mathcal{D}=\mathcal{X}$, it is well-known that (44) results in a sequence of nested sets that approximate the maximal $\lambda$-contractive set $\mathcal{C}_{\max }^{\lambda}$ (see Def. 11) from outside, i.e.,

$$
\mathcal{C}_{\max }^{\lambda} \subseteq \mathcal{Q}_{k+1}^{\lambda}(\mathcal{X}) \subseteq \mathcal{Q}_{k}^{\lambda}(\mathcal{X})
$$

for every $k \in \mathbb{N}$ (see [2, Thm. 3.1]). Moreover, if $\mathcal{C}_{\max }^{\lambda}$ is a C-set (see Def. 2), which is the case if $\lambda$ is such that $0 \in \operatorname{int}\left(\mathcal{C}_{\max }^{\lambda}\right)$ [2, Rem. 4.1], the sequence $\left\{\mathcal{Q}_{k}^{\lambda}(\mathcal{X})\right\}$ converges to $\mathcal{C}_{\max }^{\lambda}$ in the sense that, for every $\epsilon>0$, there exists a $k \in \mathbb{N}$ such that

$$
\mathcal{Q}_{k}^{\lambda}(\mathcal{X}) \subseteq(1+\epsilon) \mathcal{C}_{\max }^{\lambda}
$$

While this characteristic is, in principle, well-understood, there does (except for $\lambda=1$ ) not exist a method to a priori compute an upper bound for a suitable $k$ such that (6) holds for a given $\epsilon$. In this paper, we derive such a bound by adapting and extending related results on the convergence of null-controllable sets given in [4].

The presented bound on $k$ allows the solution of another problem related to the computation of $\lambda$-contractive sets. It is well-known that $\lambda$-contractive sets can be used to approximate the maximal controlled invariant set $\mathcal{C}_{\max }^{1}$ arbitrarily closely (consider, e.g., [7, Lem. 2.1 and Thm. 2.1]). However, it is not clear how to a priori choose $\lambda \in(0,1)$ such that the relation

$$
\mu \mathcal{C}_{\max }^{1} \subseteq \mathcal{C}_{\max }^{\lambda}
$$

is guaranteed to hold for a given $\mu \in(0,1)$. We show how such a $\lambda$ can be computed before evaluating (or approximating) $\mathcal{C}_{\max }^{1}$ or $\mathcal{C}_{\max }^{\lambda}$.

\section{Notation and Preliminaries}

We begin by formalizing the notion of $\lambda$-contractive sets, controlled invariant sets, and C-sets. As a preparation, note that the scaling $\mu \mathcal{C}$ is understood as $\mu \mathcal{C}:=\{\mu x \mid x \in \mathcal{C}\}$ for any scalar $\mu>0$ and any set $\mathcal{C} \subset \mathbb{R}^{n}$.

Definition 1. Let $\lambda \in(0,1]$. A set $\mathcal{C} \subseteq \mathcal{X}$ is called $\lambda$-contractive for (11) w.r.t. (2), if for every $x \in \mathcal{C}$ there exists $u \in \mathcal{U}$ such that $A x+B u \in \lambda \mathcal{C}$. For the special case $\lambda=1$, a 1-contractive set is also called controlled invariant. For a given $\lambda$, the maximal $\lambda$-contractive set (for (11) w.r.t. (2) ), i.e., the union of all $\lambda$-contractive sets for (1) w.r.t. (2), is denoted by $\mathcal{C}_{\max }^{\lambda}$.

Definition 2. A set $\mathcal{C} \subset \mathbb{R}^{n}$ is called $C$-set if it is convex and compact and contains the origin as an interior point.

For two given $\mathrm{C}$-sets $\mathcal{C}, \mathcal{D} \subset \mathbb{R}^{n}$, we define the distance between the sets as in 4 , Sect. 2]. Specifically, let $\mathcal{S}:=\left\{\xi \in \mathbb{R}^{n} \mid\|\xi\|_{2}=1\right\}$ denote a hypersphere in $\mathbb{R}^{n}$ and let $\rho(\xi, \mathcal{C}):=\sup \{\mu>0 \mid \mu \xi \in \mathcal{C}\}$ for any $\xi \in \mathcal{S}$. Then

$$
d(\mathcal{C}, \mathcal{D}):=\sup _{\xi \in \mathcal{S}}|\ln (\rho(\xi, \mathcal{C}))-\ln (\rho(\xi, \mathcal{D}))|
$$


provides a measure of the distance between $\mathcal{C}$ and $\mathcal{D}$. In fact, it is straightforward to show that $d: \mathcal{Y} \times \mathcal{Y} \rightarrow \mathbb{R}$ is a metric on the set $\mathcal{Y}$ of all C-sets in $\mathbb{R}^{n}$. In particular, we have $d(\mathcal{C}, \mathcal{D})=d(\mathcal{D}, \mathcal{C}) \geq 0$ and $d(\mathcal{C}, \mathcal{D}) \leq d(\mathcal{C}, \mathcal{E})+d(\mathcal{D}, \mathcal{E})$ for all $\mathcal{C}, \mathcal{D}, \mathcal{E} \in \mathcal{Y}$. Moreover, $d(\mathcal{C}, \mathcal{D})=0$ if and only if $\mathcal{C}=\mathcal{D}$. Now, according to the following lemma (which we prove in the appendix), evaluating the distance $d(\mathcal{C}, \mathcal{D})$ allows one to check relations of the form (6).

Lemma 1. Let $\delta \geq 0$ and let $\mathcal{C}, \mathcal{D} \subset \mathbb{R}^{n}$ be C-sets with $\mathcal{C} \subseteq \mathcal{D}$. Then

$$
\begin{aligned}
& \mathcal{D} \subseteq \exp (\delta) \mathcal{C} \quad \Longleftrightarrow \quad d(\mathcal{C}, \mathcal{D}) \leq \delta \quad \text { and } \\
& d(\mathcal{C}, \mathcal{D})=\min _{\mu \in \mathbb{R}} \ln (\mu) \quad \text { s.t. } \quad \mathcal{D} \subseteq \mu \mathcal{C} .
\end{aligned}
$$

Finally, we introduce the shorthand notation $\mathbb{N}_{[i, k]}:=\{j \in \mathbb{N} \mid i \leq j \leq k\}$ and we denote the smallest and largest singular values of a matrix $\Phi \in \mathbb{R}^{n \times l}$ by $\sigma_{\min }(\Phi)$ and $\sigma_{\max }(\Phi)$, respectively. We further stress that most of the results presented in this paper require the two following assumptions on the system matrices $A \in \mathbb{R}^{n \times n}$ and $B \in \mathbb{R}^{n \times m}$ and the constraint sets $\mathcal{X} \subset \mathbb{R}^{n}$ and $\mathcal{U} \subset \mathbb{R}^{m}$ to hold.

Assumption 1. The pair $(A, B)$ is controllable.

Assumption 2. The sets $\mathcal{X}$ and $\mathcal{U}$ are $C$-sets.

\section{Proof of Contraction}

The following theorem provides the key to prove the statements about $\lambda$-contractive sets mentioned in the introduction and summarized in Thms. 9 and 12 further below. Theorem 2 states that the mapping $\mathcal{Q}_{n}^{\lambda}(\mathcal{C})$, i.e., the set $\mathcal{Q}_{k}^{\lambda}(\mathcal{C})$ for $k$ equal to the state space dimension $n$, is a contraction on $(\mathcal{Y}, d)$ for every choice $\lambda \in(0,1]$. Note that Thm. [2 is similar to but different from [4, Thm. 2.3]. The most important difference is that [4, Thm. 2.3] only applies to the special case $\lambda=1$. In this paper, however, we are especially interested in cases where $\lambda \in(0,1)$. Moreover, [4, Thm. 2.3] requires $A$ to be invertible (see, for instance, 4, Eq. (2.4)], the computation of $\delta$ in the proof of [4, Thm. 2.3], or [5, Assum. 2.8]). This technical restriction is not necessary to show Thm. 2,

Theorem 2. Let $\lambda \in(0,1]$ and let Assums. 1 and 0 be satisfied. Then, there exists an $\eta \in[0,1)$, depending only on the system matrices $A$ and $B$, the constraints $\mathcal{X}$ and $\mathcal{U}$, and the contraction $\lambda$, such that

$$
d\left(\mathcal{Q}_{n}^{\lambda}(\mathcal{C}), \mathcal{Q}_{n}^{\lambda}(\mathcal{D})\right) \leq \eta d(\mathcal{C}, \mathcal{D})
$$

for all $C$-sets $\mathcal{C}, \mathcal{D} \subset \mathbb{R}^{n}$ with $\mathcal{C} \subseteq \mathcal{D}$.

The proof of Thm. 2 requires some preparation. First note that $\rho(\xi, \cdot)$ and $d(\cdot, \cdot)$ are well-defined only for C-sets. Thus, the following observation is elementary to analyze (11). 
Lemma 3. Let $\lambda \in(0,1]$, let $\mathcal{C} \subset \mathbb{R}^{n}$ be a $C$-set, and let Assum. Q be satisfied. Then $\mathcal{Q}_{k}^{\lambda}(\mathcal{C})$ is a $C$-set for every $k \in \mathbb{N}$.

The proof of Lem. 3 is well-known. See for example [2, Props. 3.1 and 3.2] and [4, Rem. 2.2]. Moreover, it is trivial to show (by induction) that the following relation holds.

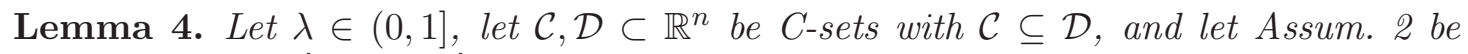
satisfied. Then $\mathcal{Q}_{k}^{\lambda}(\mathcal{C}) \subseteq \mathcal{Q}_{k}^{\lambda}(\mathcal{D})$ for every $k \in \mathbb{N}$.

Finally, it will be useful to state (necessary and sufficient) conditions for a state $x \in \mathbb{R}^{n}$ to be contained in the set $\mathcal{Q}_{k}^{\lambda}(\mathcal{C})\left(\right.$ resp. $\mathcal{Q}_{k+1}^{\lambda}(\mathcal{C})$ ). This is done in the following lemma.

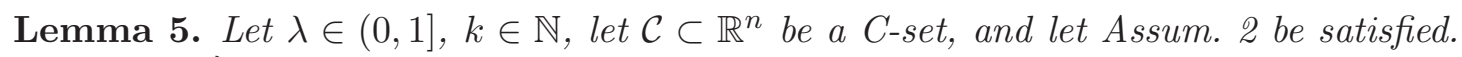
Then $x \in \mathcal{Q}_{k+1}^{\lambda}(\mathcal{C})$ if and only if there exist $u_{0}, \ldots, u_{k} \in \mathcal{U}$ and $\gamma \in \mathcal{C}$ such that

$$
\begin{aligned}
& A^{j} x+\sum_{i=0}^{j-1} A^{j-1-i} B \lambda^{i} u_{i} \in \lambda^{j} \mathcal{X} \quad \forall j \in \mathbb{N}_{[0, k]} \quad \text { and } \\
& A^{k+1} x+\sum_{i=0}^{k} A^{k-i} B \lambda^{i} u_{i}=\lambda^{k+1} \gamma
\end{aligned}
$$

Proof. Consider any C-set $\mathcal{T} \subset \mathbb{R}^{n}$ and note that $x \in \mathcal{Q}_{1}^{\lambda}(\mathcal{T})$ if and only if (i) $x \in \mathcal{X}$ and (ii) there exist $u \in \mathcal{U}$ and $\tau \in \mathcal{T}$ such that $\lambda \tau=A x+B u$. Consequently, $x \in$ $\mathcal{Q}_{k+1}^{\lambda}(\mathcal{C})=\mathcal{Q}_{1}^{\lambda}\left(\mathcal{Q}_{k}^{\lambda}(\mathcal{C})\right)$ if and only if (i) $x \in \mathcal{X}$ and (ii) there exist $u_{0} \in \mathcal{U}$ and $q_{k} \in \mathcal{Q}_{k}^{\lambda}(\mathcal{C})$ such that $\lambda q_{k}=A x+B u_{0}$. By the same reasoning, $q_{k} \in \mathcal{Q}_{k}^{\lambda}(\mathcal{C})$ if and only if (i) $q_{k} \in \mathcal{X}$ and (ii) there exist $u_{1} \in \mathcal{U}$ and $q_{k-1} \in \mathcal{Q}_{k-1}^{\lambda}(\mathcal{C})$ such that $\lambda q_{k-1}=A q_{k}+B u_{1}$. Finally, $q_{1} \in \mathcal{Q}_{1}^{\lambda}(\mathcal{C})$ if and only if (i) $q_{1} \in \mathcal{X}$ and (ii) there exist $u_{k} \in \mathcal{U}$ and $q_{0} \in \mathcal{Q}_{0}^{\lambda}(\mathcal{C})=\mathcal{C}$ such that $\lambda q_{0}=A q_{1}+B u_{k}$. The conditions (12) and (13) are obtained by collecting the the conditions (i) and (ii), and defining $\gamma:=q_{0}$.

Lemmas 3, 4, and 5 allow to prove Thm. 2,

Proof of Thm. Q The proof consists of three parts. In part (i), we derive a useful upper bound for $d\left(\mathcal{Q}_{n}^{\lambda}(\mathcal{C}), \mathcal{Q}_{n}^{\lambda}(\mathcal{D})\right)$. In part (ii), we identify some special states $x^{*}$ that are guaranteed to be contained in $\mathcal{Q}_{n}^{\lambda}(\mathcal{C})$. These states are finally used to construct an $\eta \in[0,1$ ) satisfying (11) in part (iii) of the proof.

Part (i). Consider any C-sets $\mathcal{C}, \mathcal{D} \subset \mathbb{R}^{n}$ with $\mathcal{C} \subseteq \mathcal{D}$, let $\delta:=d(\mathcal{C}, \mathcal{D})$, and define $\mu:=\exp (\delta)$. Note that $\delta \geq 0$ and $\mu \geq 1$. Moreover, we have $\mathcal{D} \subseteq \mu \mathcal{C}$ according to Lem. 1. Based on this, we obtain $\mathcal{Q}_{n}^{\lambda}(\mathcal{C}) \subseteq \mathcal{Q}_{n}^{\lambda}(\mathcal{D}) \subseteq \mathcal{Q}_{n}^{\lambda}(\mu \mathcal{C})$ according to Lem. 4 and

$$
\rho\left(\xi, \mathcal{Q}_{n}^{\lambda}(\mathcal{C})\right) \leq \rho\left(\xi, \mathcal{Q}_{n}^{\lambda}(\mathcal{D})\right) \leq \rho\left(\xi, \mathcal{Q}_{n}^{\lambda}(\mu \mathcal{C})\right)
$$

for every $\xi \in \mathcal{S}$ by definition of $\rho(\cdot, \cdot)$. Thus, a first upper bound for the distance $d\left(\mathcal{Q}_{n}^{\lambda}(\mathcal{C}), \mathcal{Q}_{n}^{\lambda}(\mathcal{D})\right)$ reads

$$
d\left(\mathcal{Q}_{n}^{\lambda}(\mathcal{C}), \mathcal{Q}_{n}^{\lambda}(\mathcal{D})\right) \leq \sup _{\xi \in \mathcal{S}} \ln \left(\frac{\rho\left(\xi, \mathcal{Q}_{n}^{\lambda}(\mu \mathcal{C})\right)}{\rho\left(\xi, \mathcal{Q}_{n}^{\lambda}(\mathcal{C})\right)}\right) .
$$


Part (ii). In the following, let $\xi \in \mathcal{S}$ be arbitrary but fixed. Define $\varrho:=\rho\left(\xi, \mathcal{Q}_{n}^{\lambda}(\mu \mathcal{C})\right)$ and observe that $\varrho>0$. Let $x:=\varrho \xi$ and note $x \in \mathcal{Q}_{n}^{\lambda}(\mu \mathcal{C})$. Thus, according to Lem. [5, there exist $u_{0}, \ldots, u_{n-1} \in \mathcal{U}$ and $\gamma \in \mu \mathcal{C}$ such that Eqs. (12) and (13) hold for $k=n$. For the case addressed here, conditions (12) and (13) can be rewritten as follows. There exist $u_{0}, \ldots, u_{n-1} \in \mathcal{U}$ and $\gamma^{*} \in \mathcal{C}$ such that

$$
\begin{aligned}
& A^{j} \varrho \xi+\sum_{i=0}^{j-1} A^{j-1-i} B \lambda^{i} u_{i} \in \lambda^{j} \mathcal{X} \quad \forall j \in \mathbb{N}_{[0, n-1]} \quad \text { and } \\
& A^{n} \varrho \xi+\sum_{i=0}^{n-1} A^{n-1-i} B \lambda^{i} u_{i}=\mu \lambda^{n} \gamma^{*} .
\end{aligned}
$$

We now prove there also exist $v_{0}, \ldots, v_{n-1} \in \mathcal{U}$ and $\varrho$ $>0$ such that

$$
\begin{aligned}
& A^{j} \varrho \hat{\varrho}+\sum_{i=0}^{j-1} A^{j-1-i} B \lambda^{i} v_{i} \in \lambda^{j} \mathcal{X} \quad \forall j \in \mathbb{N}_{[0, n-1]} \quad \text { and } \\
& A^{n} \hat{\varrho} \xi+\sum_{i=0}^{n-1} A^{n-1-i} B \lambda^{i} v_{i}=0 .
\end{aligned}
$$

As a preparation, let $\mathcal{B}^{n}(r)$ denote a ball in $\mathbb{R}^{n}$ of radius $r$ centered at the origin. Then, since $\mathcal{X}$ and $\mathcal{U}$ are C-sets by Assum. 2, there exist $\bar{r}_{x} \geq \underline{r}_{x}>0$ and $\underline{r}_{u}>0$ such that

$$
\mathcal{B}^{n}\left(\underline{r}_{x}\right) \subseteq \mathcal{X} \subseteq \mathcal{B}^{n}\left(\bar{r}_{x}\right) \quad \text { and } \quad \mathcal{B}^{m}\left(\underline{r}_{u}\right) \subseteq \mathcal{U},
$$

which obviously implies $\sup _{\xi \in \mathcal{S}} \rho(\xi, \mathcal{X}) \leq \bar{r}_{x}$. In addition, let

$$
\alpha:=\max \left\{1, \max _{j \in \mathbb{N}_{[1, n]}}\left\|A^{j}\right\|_{2}\right\}
$$

and define

$$
\Phi_{j}:=\left(A^{j-1} B, \ldots, A^{0} B\right) \in \mathbb{R}^{n \times j m}
$$

and $\vartheta_{j}:=\left(\lambda^{0} v_{0}, \ldots, \lambda^{j-1} v_{j-1}\right)^{T} \in \mathbb{R}^{j m}$ for every $j \in \mathbb{N}_{[1, n]}$. Note that $\alpha \geq 1$ and $\sigma_{\min }\left(\Phi_{n}\right)>0$ by construction and since $\Phi_{n}$ has full rank as a consequence of Assum. 1. Consequently, the choice

$$
\hat{\varrho}=\frac{\lambda^{n-1}}{\alpha} \min \left\{\frac{\underline{r}_{x}}{1+\frac{\sigma_{\max }\left(\Phi_{n}\right)}{\sigma_{\min }\left(\Phi_{n}\right)}}, \underline{r_{u}} \sigma_{\min }\left(\Phi_{n}\right)\right\}
$$

implies $\hat{\varrho}>0$. Moreover, we have

$$
A^{j} \varrho \xi+\sum_{i=0}^{j-1} \lambda^{i} A^{j-1-i} B v_{i}=A^{j} \hat{\varrho} \xi+\Phi_{j} \vartheta_{j}
$$


for every $j \in \mathbb{N}_{[1, n]}$ by construction. We next show that, for the state $\hat{x}:=\hat{\varrho} \xi$, we can always compute $n$ inputs $v_{0}, \ldots, v_{n-1} \in \mathcal{U}$ such that (18) holds. In fact, since the Moore-Penrose pseudoinverse $\Phi_{n}^{+}:=\Phi_{n}^{T}\left(\Phi_{n} \Phi_{n}^{T}\right)^{-1}$ has full rank (again due to Assum. (1), $\vartheta_{n}=-\Phi_{n}^{+} A^{n} \varrho \hat{\varrho} \xi$ results a suitable choice. Clearly, the associated inputs $v_{0}, \ldots, v_{n-1}$ satisfy (18). Moreover, we have

$$
\left\|\vartheta_{n}\right\|_{2} \leq \hat{\varrho}\left\|\Phi_{n}^{+}\right\|_{2}\left\|A^{n}\right\|_{2}\|\xi\|_{2} \leq \frac{\alpha \hat{\varrho}}{\sigma_{\min }\left(\Phi_{n}\right)}
$$

and consequently

$$
\left\|\left(v_{0}, \ldots, v_{n-1}\right)^{T}\right\|_{2} \leq \frac{1}{\lambda^{n-1}}\left\|\vartheta_{n}\right\|_{2} \leq \frac{\hat{\varrho} \alpha}{\lambda^{n-1} \sigma_{\min }\left(\Phi_{n}\right)} \leq \underline{r}_{u},
$$

where the last relation holds because of (22). Thus, we obtain $v_{i} \in \mathcal{B}^{m}\left(\underline{r}_{u}\right) \subseteq \mathcal{U}$ for every $i \in \mathbb{N}_{[0, n-1]}$ according to (19). To show (17), first note that, for every $j \in \mathbb{N}_{[1, n-1]}$, we obtain

$$
\begin{aligned}
\left\|A^{j} \hat{\varrho} \xi+\Phi_{j} \vartheta_{j}\right\|_{2} & \leq \alpha \hat{\varrho}+\left\|\Phi_{j}\right\|_{2}\left\|\vartheta_{j}\right\|_{2} \leq \alpha \hat{\varrho}+\left\|\Phi_{n}\right\|_{2}\left\|\vartheta_{n}\right\|_{2} \\
& \leq \alpha \hat{\varrho}\left(1+\frac{\sigma_{\max }\left(\Phi_{n}\right)}{\sigma_{\min }\left(\Phi_{n}\right)}\right) \leq \lambda^{n-1} \underline{r}_{x}
\end{aligned}
$$

according to Eqs. (22)-(24) and due to $\left\|\Phi_{n}\right\|_{2}=\sigma_{\max }\left(\Phi_{n}\right)$. Thus, (17) holds for every $j \in \mathbb{N}_{[1, n-1]}$ since

$$
\mathcal{B}^{n}\left(\lambda^{n-1} \underline{r}_{x}\right)=\lambda^{n-1} \mathcal{B}^{n}\left(\underline{r}_{x}\right) \subseteq \lambda^{n-1} \mathcal{X} \subseteq \lambda^{j} \mathcal{X}
$$

follows from (19)). Clearly, (17) also holds for $j=0$ since $\varrho \xi \in \mathcal{B}^{n}\left(\lambda^{n-1} \underline{r}_{x}\right) \subseteq \lambda^{0} \mathcal{X}$ is ensured by (22). Now, combining the results from (15)-(16) and (17)-(18), we can show that there exist $w_{0}, \ldots, w_{n-1} \in \mathcal{U}$ and $\varrho^{*}>0$ such that

$$
\begin{aligned}
& A^{j} \varrho^{*} \xi+\sum_{i=0}^{j-1} A^{j-1-i} B \lambda^{i} w_{i} \in \lambda^{j} \mathcal{X} \quad \forall j \in \mathbb{N}_{[0, n-1]} \quad \text { and } \\
& A^{n} \varrho^{*} \xi+\sum_{i=0}^{n-1} A^{n-1-i} B \lambda^{i} w_{i}=\lambda^{n} \gamma^{*} .
\end{aligned}
$$

In fact, the choices

$$
w_{i}:=\frac{1}{\mu} u_{i}+\frac{\mu-1}{\mu} v_{i} \text { and } \varrho^{*}:=\frac{1}{\mu} \varrho+\frac{\mu-1}{\mu} \hat{\varrho},
$$

which satisfy $w_{i} \in \mathcal{U}$ and $\varrho^{*}>0$, allow to rewrite (25) as

$$
A^{j} \varrho \xi+\sum_{i=0}^{j-1} A^{j-1-i} B \lambda^{i} u_{i}+(\mu-1)\left(A^{j} \varrho \xi+\sum_{i=0}^{j-1} A^{j-1-i} B \lambda^{i} v_{i}\right) \in \mu \lambda^{j} \mathcal{X},
$$

which holds according to (15) and (17). Analogously, (26) can be proven using Eqs. (16), (18), and (27). Thus, due to (25) $-(26)$ and $\gamma^{*} \in \mathcal{C}$, we find $x^{*}:=\varrho^{*} \xi \in \mathcal{Q}_{n}^{\lambda}(\mathcal{C})$ according to Lem. 囵, 
Part (iii). Clearly, $\varrho^{*} \xi \in \mathcal{Q}_{n}^{\lambda}(\mathcal{C})$ implies $\varrho^{*} \leq \rho\left(\xi, \mathcal{Q}_{n}^{\lambda}(\mathcal{C})\right)$. Since $\xi \in \mathcal{S}$ was arbitrary, we obtain

$$
\frac{\rho\left(\xi, \mathcal{Q}_{n}^{\lambda}(\mu \mathcal{C})\right)+(\mu-1) \varrho \underline{\varrho}}{\mu} \leq \rho\left(\xi, \mathcal{Q}_{n}^{\lambda}(\mathcal{C})\right)
$$

for every $\xi \in \mathcal{S}$ according to (27) and by definition of $\varrho$. In addition, since $\mathcal{Q}_{n}^{\lambda}(\mu \mathcal{C}) \subseteq \mathcal{X}$ and due to (19), we have

$$
\sup _{\xi \in \mathcal{S}} \rho\left(\xi, \mathcal{Q}_{n}^{\lambda}(\mu \mathcal{C})\right) \leq \sup _{\xi \in \mathcal{S}} \rho(\xi, \mathcal{X}) \leq \bar{r}_{x}
$$

We next define $\gamma:=\frac{\hat{\varrho}}{\bar{r}_{x}}$ and note that $\gamma \in(0,0.5]$ since $\varrho \leq \frac{\underline{\underline{r}}}{2}$ according to (22) (due to $\alpha \geq 1, \sigma_{\max }\left(\Phi_{n}\right) \geq \sigma_{\min }\left(\Phi_{n}\right)>0$, and $\left.\lambda \in(0,1]\right)$. Now, combining (28) and (29), we infer

$$
\begin{aligned}
\sup _{\xi \in \mathcal{S}} \ln \left(\frac{\rho\left(\xi, \mathcal{Q}_{n}^{\lambda}(\mu \mathcal{C})\right)}{\rho\left(\xi, \mathcal{Q}_{n}^{\lambda}(\mathcal{C})\right)}\right) & \leq \sup _{\xi \in \mathcal{S}} \ln \left(\frac{\mu}{1+\frac{(\mu-1) \hat{\varrho}}{\rho\left(\xi, \mathcal{Q}_{n}^{\lambda}(\mu \mathcal{C})\right)}}\right) \leq \ln \left(\frac{\mu}{1+\frac{(\mu-1) \hat{\varrho}}{\bar{r}_{x}}}\right) \\
& =\ln (\mu)-\ln (1+(\mu-1) \gamma) \leq \ln (\mu)-\ln \left(\mu^{\gamma}\right) \\
& =(1-\gamma) \ln (\mu),
\end{aligned}
$$

where the last inequality holds since $\mu^{\gamma}$ underestimates $1+(\mu-1) \gamma$ (given that $\mu \geq 1$ and $\gamma \in(0,0.5])$. Finally, taking $\ln (\mu)=\delta=d(\mathcal{C}, \mathcal{D})$ and (14) into account, it is easy to see that (30) proves (11) for the choice $\eta:=1-\gamma=1-\frac{\hat{\varrho}}{\bar{r}_{x}}$. In fact, with regard to relations (19) -(22), this choice of $\eta$ only depends on the system matrices $A$ and $B$, the constraints $\mathcal{X}$ and $\mathcal{U}$, and the contraction $\lambda$. Moreover, we have $\eta \in[0.5,1) \subset[0,1)$ due to $\gamma \in(0,0.5]$.

Theorem 2 establishes the contraction of $\mathcal{Q}_{n}^{\lambda}(\mathcal{C})$. Using similar arguments (but omitting the variations (17) and (18) which require controllability), it is easy to prove the following weaker relation, which however holds for every $k \in \mathbb{N}$.

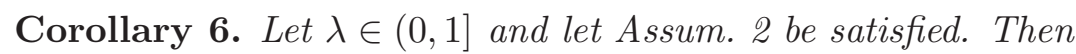

$$
d\left(\mathcal{Q}_{k}^{\lambda}(\mathcal{C}), \mathcal{Q}_{k}^{\lambda}(\mathcal{D})\right) \leq d(\mathcal{C}, \mathcal{D})
$$

for every $k \in \mathbb{N}$ and all $C$-sets $\mathcal{C}, \mathcal{D} \subset \mathbb{R}^{n}$ with $\mathcal{C} \subseteq \mathcal{D}$.

Theorem 2 and Cor. 6 lead to Lem. 8, which will be instrumental to prove Thms. 9 and 12 in the next section. As a preparation, we state the following corollary, which summarizes the choice of a suitable contraction factor $\eta$ according to the proof of Thm. 2.

Corollary 7. Let $\lambda \in(0,1]$ and let Assums. 1 and 0 be satisfied. Moreover, let $\underline{r}_{x}, \bar{r}_{x}$, and $\underline{r}_{u}$ with $0<\underline{r}_{x} \leq \bar{r}_{x}$ and $0<\underline{r}_{u}$ be such that (19) holds, define $\alpha$ and $\Phi_{n}$ according to (201) and (21), respectively, and choose $\hat{\varrho}$ as in (22). Then, $\eta=1-\frac{\hat{\varrho}}{\bar{r}_{x}}$ is such that (11) holds for all $C$-sets $\mathcal{C}, \mathcal{D} \subset \mathbb{R}^{n}$ with $\mathcal{C} \subseteq \mathcal{D}$. 
Lemma 8. Let $\lambda \in(0,1]$ and $\delta>0$, let $\mathcal{C}, \mathcal{D} \subset \mathbb{R}^{n}$ be $C$-sets with $\mathcal{C} \subseteq \mathcal{D}$, and let Assums. 1 and $⿴ 囗 0$ be satisfied. Choose $\eta$ according to Cor. 7 . Then $d\left(\mathcal{Q}_{k}^{\lambda}(\mathcal{C}), \mathcal{Q}_{k}^{\lambda}(\mathcal{D})\right) \leq \delta$ for every $k \in \mathbb{N}$ with

$$
k \geq \begin{cases}n\left\lceil\frac{\ln (\delta)-\ln (d(\mathcal{C}, \mathcal{D}))}{\ln (\eta)}\right\rceil & \text { if } d(\mathcal{C}, \mathcal{D})>\delta \\ 0 & \text { otherwise. }\end{cases}
$$

Proof. If $d(\mathcal{C}, \mathcal{D}) \leq \delta$, we find $d\left(\mathcal{Q}_{k}^{\lambda}(\mathcal{C}), \mathcal{Q}_{k}^{\lambda}(\mathcal{D})\right) \leq \delta$ for every $k \in \mathbb{N}$ according to Cor. 6 . It remains to address the case $d(\mathcal{C}, \mathcal{D})>\delta$. Theorem 2 implies $d\left(\mathcal{Q}_{j n}^{\lambda}(\mathcal{C}), \mathcal{Q}_{j n}^{\lambda}(\mathcal{D})\right) \leq$ $\eta^{j} d(\mathcal{C}, \mathcal{D})$ for every $j \in \mathbb{N}$. Clearly, $\eta^{j} d(\mathcal{C}, \mathcal{D}) \leq \delta$ if

$$
j \geq \frac{\ln (\delta)-\ln (d(\mathcal{C}, \mathcal{D})}{\ln (\eta)} .
$$

Taking $j \in \mathbb{N}$ into account leads to (32).

\section{Implications}

In this section, we provide formal proofs for the new results on $\lambda$-contractive sets stated in the introduction and summarized in Thms. 9 and 12 .

Theorem 9. Let $\lambda \in(0,1]$ and $\epsilon>0$, let Assums. 1 and 0 be satisfied, and let $\mathcal{C}$ be any $\lambda$-contractive $C$-set. Set $\mathcal{D}=\mathcal{X}$ and $\delta=\ln (1+\epsilon)$, choose $\eta$ according to Cor. 7 , and let $k$ be such that (32) holds. Then

$$
\mathcal{Q}_{k}^{\lambda}(\mathcal{X}) \subseteq(1+\epsilon) \mathcal{Q}_{k}^{\lambda}(\mathcal{C}) \subseteq(1+\epsilon) \mathcal{C}_{\max }^{\lambda}
$$

Proof. Since $\mathcal{C}$ is $\lambda$-contractive, it is easy to show that $\mathcal{Q}_{k}^{\lambda}(\mathcal{C})$ is $\lambda$-contractive for every $k \in \mathbb{N}$. Thus, we have $\mathcal{Q}_{k}^{\lambda}(\mathcal{C}) \subseteq \mathcal{C}_{\max }^{\lambda}$ for every $k \in \mathbb{N}$, which proves the second relation in (33). For a $k$ satisfying (32), we obtain $d\left(\mathcal{Q}_{k}^{\lambda}(\mathcal{C}), \mathcal{Q}_{k}^{\lambda}(\mathcal{X})\right) \leq \delta$ according to Lem. 8 . Hence, the first relation in (33) holds according to Lem. 1 1 and due to the choice of $\delta$.

Remark 1. As mentioned in the introduction, existence of a $k \in \mathbb{N}$ satisfying (6) for given $\lambda$ and $\epsilon$ requires $\mathcal{C}_{\max }^{\lambda}$ to be a C-set. A necessary and sufficient condition for $\mathcal{C}_{\max }^{\lambda}$ to be a $C$-set is the existence of some $\lambda$-contractive $C$-set $\mathcal{C}$. Clearly, the explicit knowledge of such a set $\mathcal{C}$, as required in Thm. [. is more restrictive than the fundamental assumption that $\mathcal{C}_{\max }^{\lambda}$ is a $C$-set. However, there exist a number of procedures to identify (small) $\lambda$-contractive $C$-sets. Assume, for example, there exist $K \in \mathbb{R}^{m \times n}$ and a positive definite matrix $P \in \mathbb{R}^{n \times n}$ such that $(A+B K)^{T} P(A+B K) \preceq \lambda^{2} P$ and such that $A+B K$ is Schur stable. Now choose any $\beta>0$ such that $\mathcal{C}=\left\{x \in \mathbb{R}^{n} \mid x^{T} P x \leq \beta\right\} \subseteq\{x \in$ $\mathcal{X} \mid K x \in \mathcal{U}\}$. Then, $\mathcal{C}$ is a $\lambda$-contractive ellipsoid and thus a $\lambda$-contractive $C$-set (cf. [2, Rem. 4.1]). Similar procedures exist to compute $\lambda$-contractive polytopes (see, e.g., [8, Sect. V]).

Theorem 12 further below addresses the suitable choice of $\lambda \in(0,1)$ to guarantee (7) for a given $\mu \in(0,1)$. As a preparation, we provide the following two lemmas. 


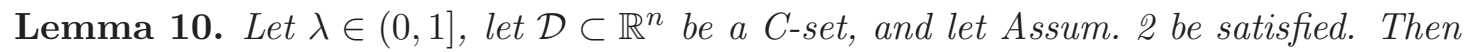
$\lambda^{k} \mathcal{Q}_{k}^{1}(\mathcal{D}) \subseteq \mathcal{Q}_{k}^{\lambda}(\mathcal{D})$ for every $k \in \mathbb{N}$.

Proof. The relation holds with equality for $k=0$. We prove the relation for $k>0$ by induction. First note that, for any C-set $\mathcal{T} \subset \mathbb{R}^{n}, \mathcal{Q}_{1}^{\lambda}(\mathcal{T})$ can be written as

$$
\mathcal{Q}_{1}^{\lambda}(\mathcal{T})=\lambda\left\{\tilde{x} \in \lambda^{-1} \mathcal{X} \mid \exists \tilde{u} \in \lambda^{-1} \mathcal{U}: A \tilde{x}+B \tilde{u} \in \mathcal{T}\right\}
$$

Since $\lambda \leq 1$ implies $\lambda^{-1} \mathcal{X} \supseteq \mathcal{X}$ and $\lambda^{-1} \mathcal{U} \supseteq \mathcal{U}$, we obtain $\lambda \mathcal{Q}_{1}^{1}(\mathcal{T}) \subseteq \mathcal{Q}_{1}^{\lambda}(\mathcal{T})$. To show that $\lambda^{k} \mathcal{Q}_{k}^{1}(\mathcal{D}) \subseteq \mathcal{Q}_{k}^{\lambda}(\mathcal{D})$ implies $\lambda^{k+1} \mathcal{Q}_{k+1}^{1}(\mathcal{D}) \subseteq \mathcal{Q}_{k+1}^{\lambda}(\mathcal{D})$, first note that

$$
\mathcal{Q}_{k+1}^{\lambda}(\mathcal{D})=\mathcal{Q}_{1}^{\lambda}\left(\mathcal{Q}_{k}^{\lambda}(\mathcal{D})\right) \supseteq \mathcal{Q}_{1}^{\lambda}\left(\lambda^{k} \mathcal{Q}_{k}^{1}(\mathcal{D})\right) \supseteq \lambda \mathcal{Q}_{1}^{1}\left(\lambda^{k} \mathcal{Q}_{k}^{1}(\mathcal{D})\right) .
$$

Now, rewriting $\mathcal{Q}_{1}^{1}(\mu \mathcal{T})$ in the style of (34) for some $\mu \in(0,1]$, it is easy to show that $\mu \mathcal{Q}_{1}^{1}(\mathcal{T}) \subseteq \mathcal{Q}_{1}^{1}(\mu \mathcal{T})$. We consequently find $\lambda^{k} \mathcal{Q}_{1}^{1}(\mathcal{T}) \subseteq \mathcal{Q}_{1}^{1}\left(\lambda^{k} \mathcal{T}\right)$. Taking (35) into account, we finally infer

$$
\mathcal{Q}_{k+1}^{\lambda}(\mathcal{D}) \supseteq \lambda^{k+1} \mathcal{Q}_{1}^{1}\left(\mathcal{Q}_{k}^{1}(\mathcal{D})\right)=\lambda^{k+1} \mathcal{Q}_{k+1}^{1}(\mathcal{D}),
$$

which completes the proof.

Lemma 11. Let $\mu \in(0,1)$ and $\lambda^{*} \in(0,1)$, let Assums. 1 and Q be satisfied, let $\mathcal{C}$ be $a \lambda^{*}$-contractive $C$-set, and set $\epsilon=\frac{1-\mu}{2 \mu}$. Then, there exist $\lambda \in\left[\lambda^{*}, 1\right)$ and $k \in \mathbb{N}$ such that (33) holds and such that

$$
1+\mu \leq 2 \lambda^{k}
$$

Proof. Set $\lambda=\lambda^{*}, \mathcal{D}=\mathcal{X}$, and $\delta=\ln (1+\epsilon)$ and let $\underline{r}_{x}, \bar{r}_{x}, \underline{r}_{u}, \alpha$ and $\Phi_{n}$ be as in Cor. (7. Choose $\varrho$ as in (22), set $\eta=1-\frac{\hat{\varrho}}{\bar{r}_{x}}$, and pick any $k \in \mathbb{N}$ that satisfies (32). Then, relation (33) holds according to Thm. 9. However, we either have (i) $1+\mu \leq 2\left(\lambda^{*}\right)^{k}$ or (ii) $1+\mu>2\left(\lambda^{*}\right)^{k}$. Case (i) immediately finishes the proof.

In contrast, if case (ii) applies, first note that we have $k>0\left(\right.$ since $1+\mu>2\left(\lambda^{*}\right)^{0}=2$ contradicts $\mu<1$ ). Now, compute

$$
\lambda=\exp \left(\frac{\ln (1+\mu)-\ln (2)}{k}\right)
$$

and note that $2 \lambda^{k}=1+\mu$ and $\lambda \in\left(\lambda^{*}, 1\right)$. Clearly, since $\lambda>\lambda^{*}$, the set $\mathcal{C}$ is also $\lambda$-contractive. Now, recompute $\hat{\varrho}$ according to (22) for the new value of $\lambda$ given by (37). Note that the new $\hat{\varrho}$ is larger than the one that was obtained above with $\lambda=\lambda^{*}$. Consequently, the recalculation of $\eta=1-\frac{\hat{\rho}}{\bar{r}_{x}}$ results in a smaller value than above. Thus, it is easy to see that $k$ as chosen above still satisfies (32). This completes the proof, since (33) again holds according to Thm. 9 and since (36) is satisfied by construction.

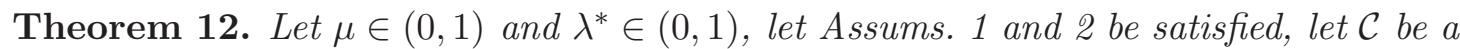
$\lambda^{*}$-contractive $C$-set, and set $\epsilon=\frac{1-\mu}{2 \mu}$. Assume $\lambda \in\left[\lambda^{*}, 1\right)$ and $k \in \mathbb{N}$ are such that (33) and (36) hold. Then

$$
\mu \mathcal{C}_{\max }^{1} \subseteq \mathcal{Q}_{k}^{\lambda}(\mathcal{C}) \subseteq \mathcal{C}_{\max }^{\lambda}
$$


Proof. We have $\mathcal{C}_{\max }^{1} \subseteq \mathcal{Q}_{k}^{1}(\mathcal{X})$ and $\lambda^{k} \mathcal{Q}_{k}^{1}(\mathcal{X}) \subseteq \mathcal{Q}_{k}^{\lambda}(\mathcal{X})$ according to Eq. (15) and Lem. 10, respectively. Combining both relations and taking Eq. (33) into account, yields

$$
\lambda^{k} \mathcal{C}_{\max }^{1} \subseteq \lambda^{k} \mathcal{Q}_{k}^{1}(\mathcal{X}) \subseteq \mathcal{Q}_{k}^{\lambda}(\mathcal{X}) \subseteq(1+\epsilon) \mathcal{Q}_{k}^{\lambda}(\mathcal{C}) \subseteq(1+\epsilon) \mathcal{C}_{\max }^{\lambda}
$$

This proves (38) since we have

$$
\mu \mathcal{C}_{\max }^{1} \subseteq \frac{2 \lambda^{k}}{1+\mu} \mu \mathcal{C}_{\max }^{1}=\frac{\lambda^{k}}{1+\epsilon} \mathcal{C}_{\max }^{1}
$$

due to (36) and by definition of $\epsilon$, respectively.

Remark 2. For the interpretation of Lem. 11 and Thm. 12, it is important to note that, for a given $\mu \in(0,1)$, suitable $\lambda \in\left[\lambda^{*}, 1\right)$ and $k \in \mathbb{N}$ satisfying (33) and (36) can be computed without evaluating the sets $\mathcal{Q}_{k}^{\lambda}(\mathcal{X}), \mathcal{Q}_{k}^{\lambda}(\mathcal{C})$, or $\mathcal{C}_{\max }^{\lambda}$ in (33). In fact, we only require the computation of (i) a $\lambda^{*}$-contractive $C$-set $\mathcal{C}$, (ii) the distance $d(\mathcal{C}, \mathcal{X})($ e.g., according to (10), and (iii) $\eta$ as in Cor. 7 . Then, suitable $\lambda$ and $k$ can be calculated according to the proof of Lem. 11.

Remark 3. For practical applications, having the guarantee that (38) holds without actually knowing (an approximation of) $\mathcal{C}_{\max }^{\lambda}$ is usually useless. Fortunately, Thm. 12 implicitly provides two methods to compute $\lambda$-contractive sets that approximate the maximal controlled invariant set $\mathcal{C}_{\max }^{1}$ with a given accuracy $\mu \in(0,1)$, presupposing that a $\lambda^{*}$-contractive set $\mathcal{C}$ is known. To see this, first note that any $\lambda \in\left[\lambda^{*}, 1\right)$ and $k \in \mathbb{N}$ satisfying (33) and (36) for $\epsilon=\frac{1-\mu}{2 \mu}$ result in a $\lambda$-contractive set $\mathcal{T}=\mathcal{Q}_{k}^{\lambda}(\mathcal{C})$ satisfying $\mu \mathcal{C}_{\max }^{1} \subseteq \mathcal{T}$. Now, a suitable set $\mathcal{T}$ can be computed using two strategies. First, we can compute $\lambda$ and $k$ according to the proof of Lem. 11 and simply evaluate $\mathcal{Q}_{k}^{\lambda}(\mathcal{C})$ according to (4). This, however, might be numerically expensive since the computed $k$ is usually quite conservative. Second, for $\lambda$ as above, we can compute $\mathcal{Q}_{j}^{\lambda}(\mathcal{C})$ and $\mathcal{Q}_{j}^{\lambda}(\mathcal{X})$ for increasing $j \in \mathbb{N}$ until $\mathcal{Q}_{j}^{\lambda}(\mathcal{X}) \subseteq(1+\epsilon) \mathcal{Q}_{j}^{\lambda}(\mathcal{C})$ is observed for $j=k^{*}$. We obviously have $k^{*} \leq k$ by construction and thus $1+\mu \leq 2 \lambda^{k} \leq 2 \lambda^{k^{*}}$. Consequently, (33) and (36) hold and $\mathcal{T}=\mathcal{Q}_{k^{*}}^{\lambda}(\mathcal{C})$ approximates $\mathcal{C}_{\max }^{1}$ accurately. The second strategy is numerically attractive, since $k^{*}$ is usually significantly smaller than $k$ from the proof of Lem. 11] (see the example in Sect. 5.2).

\section{Illustrative examples}

We analyze three examples to discuss the uses and limitations of Thms. 9 and 12 as well as Thm. 2. In particular, we show how to compute suitable $k$ and $\lambda$ such that (6) and (7) hold without evaluating $\mathcal{Q}_{k}^{\lambda}(\mathcal{X}), \mathcal{C}_{\max }^{\lambda}$, or $\mathcal{C}_{\max }^{1}$. These numbers are then compared to the optimal (i.e., smallest possible) choices of $k$ and $\lambda$. It is easy to see that the optimal choices require the knowledge of the sets $\mathcal{Q}_{k}^{\lambda}(\mathcal{X}), \mathcal{C}_{\max }^{\lambda}$, or $\mathcal{C}_{\max }^{1}$. Since explicit descriptions of these sets are usually not available for complex systems, we consider relatively simple examples in the following. We stress, however, that the techniques in Thms. 9 and 12 can be applied to more complex systems provided the requirements in Rem. 2 can be satisfied. 


\subsection{Discussion of Theorem 9}

Theorem 9 makes it possible to compute an iteration bound $k$ such that (33) holds. In the following, we compare the provided bound with the smallest $k$ satisfying (33) for a simple example.

Consider system (11) with $A=1.1 I_{n}$ and $B=I_{n}$ and constraints $\mathcal{X}=[-10,10]^{n}$ and $\mathcal{U}=[-1,1]^{n}$ for an arbitrary $n \in \mathbb{N}$ with $n>0$, where $I_{n}$ denotes the identity matrix in $\mathbb{R}^{n \times n}$. Obviously, the system can be resolved into $n$ independent systems of dimension one. Nevertheless, the conglomerated system is useful to analyze Thm. 9, In this context, first note that the set $\mathcal{C}=[-2,2]^{n}$ is $\lambda$-contractive for every $\lambda \in[0.6,1]$. Moreover, it is easy to show that the maximal $\lambda$-contractive set is given by

$$
\mathcal{C}_{\max }^{\lambda}=\left[-c_{\max }^{\lambda}, c_{\max }^{\lambda}\right]^{n} \quad \text { with } \quad c_{\max }^{\lambda}:=\frac{1}{1.1-\lambda}
$$

for this example. We obviously have $\mathcal{C}_{\max }^{\lambda}=\mathcal{C}$ for $\lambda=0.6$ and $C_{\max }^{\lambda}=\mathcal{X}$ for $\lambda=1$. Now, according to Thm. 9, for every $\lambda \in[0.6,1]$ and every $\epsilon>0$, there exists a $k \in \mathbb{N}$ such that (33) holds. Following Thm. 9, such a $k$ can be found by setting $\mathcal{D}=\mathcal{X}$ and $\delta=\ln (1+\epsilon)$, selecting $\eta$ as in Cor. 7, and choosing $k$ such that (32) holds. To this end, first note that $\underline{r}_{x}=10, \bar{r}_{x}=10 \sqrt{n}$, and $\underline{r}_{u}=1$ are such that (19) holds. Moreover, $\alpha=1.1^{n}$ satisfies (20) and it is straightforward to show that

$$
\sigma_{\max }\left(\Phi_{n}\right)=\sigma_{\min }\left(\Phi_{n}\right)=\sqrt{\sum_{i=0}^{n-1} 1.1^{2 i}}=\sqrt{\frac{1.21^{n}-1}{0.21}} .
$$

Thus, according to (22), $\underline{\varrho}$ evaluates to

$$
\hat{\varrho}=\frac{\lambda^{n-1}}{1.1^{n}} \min \left\{5, \sqrt{\frac{1.21^{n}-1}{0.21}}\right\} .
$$

Consequently, a suitable choice for $\eta$ is

$$
\eta=1-\frac{\hat{\varrho}}{\bar{r}_{x}}=1-\frac{\lambda^{n-1}}{10 \sqrt{n} 1.1^{n}} \min \left\{5, \sqrt{\frac{1.21^{n}-1}{0.21}}\right\} .
$$

It remains to choose $k$ satisfying (32). Evaluating the distance between $\mathcal{C}$ and $\mathcal{X}$ according to (10) results in $d(\mathcal{C}, \mathcal{X})=\ln (5)$. Thus, the smallest $k$ that satisfies (32) for any $\epsilon \in(0,4)$ can be computed according to

$$
k=n\left\lceil\frac{\ln (\ln (1+\epsilon))-\ln (\ln (5))}{\ln (\eta)}\right\rceil
$$

with $\eta$ as in (40). Numerical values for $n \in\{1,2\}, \lambda \in\{0.6,0.8,1.0\}$, and $\epsilon \in$ $\{0.01,0.05,0.1\}$ are listed in Tab. 1. (a).

We next compare the results in Tab. 1,(a) with the smallest $k$ such that (33) holds. For this simple example, $\mathcal{Q}_{k}^{\lambda}(\mathcal{C})$ and $\mathcal{Q}_{k}^{\lambda}(\mathcal{X})$ can be stated explicitly as

$$
\mathcal{Q}_{k}^{\lambda}(\mathcal{C})=\left[-c_{k}^{\lambda}, c_{k}^{\lambda}\right]^{n} \text { with } c_{k}^{\lambda}:=2\left(\frac{\lambda}{1.1}\right)^{k}+\frac{1-\left(\frac{\lambda}{1.1}\right)^{k}}{1.1-\lambda}
$$


and

$$
\mathcal{Q}_{k}^{\lambda}(\mathcal{X})=\left[-x_{k}^{\lambda}, x_{k}^{\lambda}\right]^{n} \text { with } x_{k}^{\lambda}:=10\left(\frac{\lambda}{1.1}\right)^{k}+\frac{1-\left(\frac{\lambda}{1.1}\right)^{k}}{1.1-\lambda}
$$

for every $k \in \mathbb{N}$ and any $\lambda \in[0.6,1]$. Now, condition (33) obviously holds for a given $k \in \mathbb{N}$ if (and only if)

$$
x_{k}^{\lambda} \leq(1+\epsilon) c_{k}^{\lambda} .
$$

Clearly, (44) does not depend on $n$. In other words, the smallest $k$ such that (33) holds does not change with $n$ for this example. Apparently, this observation is not echoed in Tab. 1.(a) (or Eq. (41)), where we clearly have a dependence on $n$. Now, based on (43) and (42), it is easy to prove that the smallest $k$ satisfying (44) reads

$$
k=\left\lceil\frac{\ln \left((1.1-\lambda)\left(\frac{8}{\epsilon}-2\right)+1\right)}{\ln (1.1)-\ln (\lambda)}\right\rceil
$$

for a given $\lambda \in[0.6,1]$ and $\epsilon \in(0,4]$. In Tab. 1,(b) numerical values for $k$ as in (45) are listed for $\lambda$ and $\epsilon$ as above. Comparing the entries in Tabs. 1.(a) and 1,(b), it turns out that the values for $k$ computed according to Thm. 9 are valid but conservative. In fact, the smallest overestimation, which is by a factor of $\frac{54}{47} \approx 1.1489$, occurs for $n=1$, $\lambda=1.0$, and $\epsilon=0.01$.

Table 1: Numerical values for $k$ as in (41) and (45), respectively, as a function of $n, \lambda$, and $\epsilon$.

(a) values for $k$ as in (41)

\begin{tabular}{rrrrr}
\hline$n$ & $\lambda$ & \multicolumn{3}{c}{$\epsilon$} \\
\cline { 3 - 5 } & & 0.01 & 0.05 & 0.1 \\
\hline 2 & 0.6 & 192 & 132 & 106 \\
2 & 0.8 & 142 & 98 & 80 \\
2 & 1.0 & 112 & 78 & 64 \\
\cline { 1 - 2 } 1 & any & 54 & 37 & 30 \\
\hline
\end{tabular}

(b) values for $k$ as in (45)

\begin{tabular}{crrr}
\hline$\lambda$ & \multicolumn{3}{c}{$\epsilon$} \\
\cline { 2 - 4 }$(\operatorname{any} n)$ & 0.01 & 0.05 & 0.1 \\
\hline 0.6 & 10 & 8 & 7 \\
0.8 & 18 & 13 & 11 \\
1.0 & 47 & 30 & 23 \\
& & & \\
\hline
\end{tabular}

Another observation is also interesting. In Tab. 1,(b), for fixed $\epsilon$, the values of $k$ increase with increasing $\lambda$. In contrast, for $n=2$ and fixed $\epsilon$, the values of $k$ in Tab. 1. (a) decrease with increasing $\lambda$. In general, for some $\lambda^{*} \leq \lambda$ and $\mathcal{D} \subseteq \mathcal{X}$, we have $\mathcal{C}_{\max }^{\lambda^{*}} \subseteq \mathcal{C}_{\max }^{\lambda}$ and $Q_{k}^{\lambda^{*}}(\mathcal{D}) \subseteq Q_{k}^{\lambda}(\mathcal{D})$ for every $k \in \mathbb{N}$. In other words, larger values of $\lambda$ imply a larger set $\mathcal{C}_{\max }^{\lambda}$, slower contraction of $\left\{Q_{k}^{\lambda}(\mathcal{X})\right\}$, and faster expansion of $\left\{Q_{k}^{\lambda}(\mathcal{C})\right\}$. Clearly, this observation does not allow a general statement about the dependence of the smallest $k$, such that (33) holds, on $\lambda$. In fact, depending on the example, we may observe a behavior similar or opposite to Tab. 1.(b) (i.e., $k$ increases or decreases with $\lambda$ ). In contrast, the iteration bound $k$ considered in Thm. 9 will always decrease with $\lambda$ (as apparent from Tab. 1.(a)). This behavior can be explained with regard to Cor. 7 and 
Lem. 8. Clearly, for larger $\lambda$, $\varrho$ as in (22) will be larger, which results in smaller $\eta=1-\frac{\hat{\varrho}}{\bar{r}_{x}}$ and finally smaller $k$ satisfying (32). While this behavior may be conservative (as it is for this example), it is required to prove Thm. 12 and the underlying Lem. 11. Indeed, the strategy to handle case (ii) in the proof of Lem. 111 builds on the fact that the computed $\eta$ for some $\lambda \in\left(\lambda^{*}, 1\right]$ is smaller than the one for $\lambda=\lambda^{*}$.

\subsection{Discussion of Theorem 12}

Theorem 12 allows $\lambda$ to be chosen such that (17) is guaranteed to hold for a given $\mu$. In the following, we compare the smallest value of $\lambda$ such that (7) holds with the value that is obtained using Lem. 11 for the example from Sect. 5.1 and $n=1$.

Assume we want to satisfy (7) for $\mu=\frac{5}{6}$. Before applying Thm. 12 (and Lem. 11), first note that the maximal controlled invariant set is $\mathcal{C}_{\max }^{1}=[-10,10]$ (according to (39) with $\lambda=1$ ). Obviously, $\mu \mathcal{C}_{\max }^{1} \subseteq \mathcal{C}_{\max }^{\lambda}$ requires

$$
10 \mu=\frac{50}{6} \leq \frac{1}{1.1-\lambda} \quad \text { or, equivalently, } \quad \frac{49}{50} \leq \lambda .
$$

Thus, $\lambda^{*}=0.98$ is the smallest choice for $\lambda$ such that (7) holds.

The computation of a suitable $\lambda$ according to Thm.12 (and Lem. 11) involves finding $\lambda$ and $k$ such that (33) and (36) hold for the choice

$$
\epsilon=\frac{1-\mu}{2 \mu}=\frac{1}{10}=0.1 \text {. }
$$

In this context, Thm. 12 and Lem. 11 require the knowledge of a $\lambda^{*}$-contractive set $\mathcal{C}$. We again consider the set $\mathcal{C}=[-2,2]$ from Sect. 5.1, which is $\lambda$-contractive for every $\lambda \in[0.6,1]$. We use $\lambda^{*}=0.98$ from above as an initial guess for the computation of a suitable $\lambda$ corresponding to the proof of Lem. 11. In other words, we first analyze whether the presented procedure is capable of identifying whether $\lambda^{*}$ is suitable. Clearly, the smallest $k$ satisfying (32) for $\mathcal{D}=\mathcal{X}$ and $\delta=\ln (1+\epsilon)$ can be computed analogously to Sect. 5.1. Hence, evaluating (41) for $n=1, \epsilon=0.1$, and $\eta$ from (40) yields $k=30$ (as itemized in Tab. 1.(a)). We obtain

$$
1+\mu \approx 1.8333>1.0910 \approx 2 \cdot 0.98^{30}=2\left(\lambda^{*}\right)^{k},
$$

i.e., (36) does not hold for the choice $\lambda=\lambda^{*}$ and we have to address case (ii) in the proof of Lem. 11. Consequently, updating $\lambda$ according to (37) yields $\lambda \approx 0.9971$. Following the argumentation in the proof of Lem. 11, the updated $\lambda$ and $k=30$ are such that (33) and (36) hold. Thus, according to Thm. 12, the updated $\lambda$ is such that (7) holds. The computed $\lambda$ is conservative in the sense that

$$
\frac{\lambda-\lambda^{*}}{1-\lambda^{*}}=0.8552=85.52 \%
$$

of the "suitable interval" $[0.98,1)$ is not identified as being suitable. However, the result can also be interpreted in a different way. To this end, we compute $\lambda$-contractive sets $\mathcal{T}$ 
that accurately approximate $\mathcal{C}_{\max }^{1}$ according to the two strategies in Rem. 3, Using the first strategy, we obtain $\mathcal{T}=\mathcal{Q}_{30}^{\lambda}(\mathcal{C})$ based on the iteration bound $k=30$. The second strategy leads to an earlier termination after $k^{*}=23$ iterations (according to (45)). This observation is interesting, since any choice $\lambda \in[0.98,0.9971]$ requires at least 21 iterations to satisfy (33) with $\epsilon=0.1$. In other words, the conservatism in the choice of $\lambda$ only slightly influences the earliest satisfaction of (33).

\subsection{Discussion of Theorem 2}

Theorems 9 and 12 both build on the contraction property in Thm. 2. It thus makes sense to discuss Thm. 2 in more detail.

First, it is important to note that the contraction property in Thm. 2 only applies to the mapping $\mathcal{Q}_{n}^{\lambda}(\mathcal{C})$, where $n$ refers to the state space dimension. Initially, this seems counter-intuitive and one would expect a contraction after every step $k$. In fact, the example discussed in Sect. 5.1 (and Sect. 5.2) shows such a behavior. There exist, however, situations where a contraction indeed only appears every $n$ steps. In this context, consider system (1) with

$$
A=\left(\begin{array}{rr}
0 & 1 \\
-1 & 0
\end{array}\right) \quad \text { and } B=\left(\begin{array}{l}
0 \\
1
\end{array}\right)
$$

and constraints $\mathcal{X}=[-5,5]^{2}$ and $\mathcal{U}=[-1,1]$ (which is taken from [9, Sect. IV-B]). We will show that, for the choice $\mathcal{C}=[-1,1] \times[-1,1]$ and $\mathcal{D}=[-2,2] \times[1,1]$, we obtain

$$
\begin{aligned}
d\left(\mathcal{Q}_{2 j+1}^{\lambda}(\mathcal{C}), \mathcal{Q}_{2 j+1}^{\lambda}(\mathcal{D})\right) & =d\left(\mathcal{Q}_{2 j}^{\lambda}(\mathcal{C}), \mathcal{Q}_{2 j}^{\lambda}(\mathcal{D})\right) \\
& =\ln \left(\frac{\lambda^{2 j}}{\sum_{i=0}^{j} \lambda^{2 i}}+1\right)
\end{aligned}
$$

for every $\lambda \in(0,1]$ and every $j \in \mathbb{N}_{[0,3]}$ (for $j>3$, i.e., $k>7$, the state constraints $\mathcal{X}$ may, depending of the choice of $\lambda$, affect the shapes of $\mathcal{Q}_{k}^{\lambda}(\mathcal{C})$ and $\mathcal{Q}_{k}^{\lambda}(\mathcal{D})$ so that (47) may no longer hold). Now, according to (47) for $j=0$, we find

$$
d\left(\mathcal{Q}_{1}^{\lambda}(\mathcal{C}), \mathcal{Q}_{1}^{\lambda}(\mathcal{D})\right)=d\left(\mathcal{Q}_{0}^{\lambda}(\mathcal{C}), \mathcal{Q}_{0}^{\lambda}(\mathcal{D})\right)=d(\mathcal{C}, \mathcal{D})=\ln (2) .
$$

In other words, (31) holds for $k=1<n$ with equality (in agreement with Cor. 6) but there is no contraction in terms of the distance between the sets after one iteration. For $k=n=2$, relation (11) can, however, be easily satisfied for the choice $\eta=1-\frac{\lambda}{\sqrt{50}}$. This follows from Cor. 7 with $\underline{r}_{x}=5, \bar{r}_{x}=\sqrt{50}, \underline{r}_{u}=1, \alpha=1, \Phi_{n}=I_{2}, \sigma_{\max }\left(\Phi_{n}\right)=$ $\sigma_{\min }\left(\Phi_{n}\right)=1$, and $\varrho \underline{\varrho}=\lambda \min \left\{\frac{\underline{r}_{x}}{2}, \underline{r_{u}}\right\}=\lambda$. To prove (47), finally note that $\mathcal{Q}_{1}^{\lambda}(\mathcal{T}$ ) evaluates to

$$
\mathcal{Q}_{1}^{\lambda}(\mathcal{T})=\left[-\lambda \tau_{2}-1, \lambda \tau_{2}+1\right] \times\left[-\lambda \tau_{1}, \lambda \tau_{1}\right]
$$

for any set $\mathcal{T}=\left[-\tau_{1}, \tau_{1}\right] \times\left[-\tau_{2}, \tau_{2}\right]$ with $\tau_{1} \in(0,5]$ and $\tau_{2} \in(0,4]$. Equation (48) allows to compute $\mathcal{Q}_{k}^{\lambda}(\mathcal{C})=\mathcal{Q}_{1}^{\lambda}\left(\mathcal{Q}_{k-1}^{\lambda}(\mathcal{C})\right)$ and $\mathcal{Q}_{k}^{\lambda}(\mathcal{D})=\mathcal{Q}_{1}^{\lambda}\left(\mathcal{Q}_{k-1}^{\lambda}(\mathcal{D})\right.$ ) for every $k \in \mathbb{N}_{[1,7]}$ (for $k>7$, the conditions on $\tau_{1}$ and $\tau_{2}$ may be violated for $\mathcal{T}=\mathcal{Q}_{k-1}(\mathcal{C})$ or $\mathcal{T}=\mathcal{Q}_{k-1}(\mathcal{D})$ ). 
Afterwards, (10) can be used to evaluate the distances $d\left(\mathcal{Q}_{k}^{\lambda}(\mathcal{C}), \mathcal{Q}_{k}^{\lambda}(\mathcal{D})\right)$. Identifying relation (47) is then straightforward.

Another important limitation of Thm. 2 is that the pair $(A, B)$ has to be controllable (see Assum. 11). Clearly, it would be desirable to extend the contraction property to systems that are "only" stabilizable. However, a simple extension is not possible as the following example shows. Consider system (1) with system matrices $A=0.8$ and $B=0$ and constraints $\mathcal{X}=[-5,5]$ and $\mathcal{U}=[-1,1]$. Note that the pair $(A, B)$ is stabilizable but not controllable. We show in the following that, for the choice $\mathcal{C}=[-1,1]$ and $\mathcal{D}=[-2,2]$, we obtain

$$
d\left(\mathcal{Q}_{k}^{\lambda}(\mathcal{C}), \mathcal{Q}_{k}^{\lambda}(\mathcal{D})\right)=d(\mathcal{C}, \mathcal{D})=1
$$

for every $k \in \mathbb{N}_{[0,4]}$ and every $\lambda \in(0,1]$. Obviously, since (49) applies for $k=n=1$, relation (11) cannot hold with $\eta<1$ for some stabilizable systems. In other words, Thm. 2 can in this form not be extended to those systems. Corollary 6 , which does not require controllability of $(A, B)$, is however consistent with (49). To show (49), note that $\mathcal{Q}_{1}^{\lambda}(\mathcal{T})$ evaluates to $\mathcal{Q}_{1}^{\lambda}(\mathcal{T})=\left[-\frac{\lambda \tau}{0.8}, \frac{\lambda \tau}{0.8}\right]$ for any set $\mathcal{T}=[-\tau, \tau]$ with $\tau \in(0,4]$. Computing $\mathcal{Q}_{k}^{\lambda}(\mathcal{C})$ and $\left.\mathcal{Q}_{k}^{\lambda}(\mathcal{D})\right)$ accordingly and evaluating $d\left(\mathcal{Q}_{k}^{\lambda}(\mathcal{C}), \mathcal{Q}_{k}^{\lambda}(\mathcal{D})\right.$ as in (10) leads to (49). We finally note that (49) holds for every $k \in \mathbb{N}$ in case of $\lambda \in(0,0.8$. For $\lambda \in(0.8,1]$, however, $\mathcal{Q}_{k}^{\lambda}(\mathcal{C})$ and $\mathcal{Q}_{k}^{\lambda}(\mathcal{D})$ converge to the state constraints $\mathcal{X}$, which eventually results in violation of (49).

\section{Conclusion}

The paper presented two interesting results related to the computation of $\lambda$-contractive sets for linear constrained systems. First, we showed that it is possible to a priori compute a number of iterations $k$ that is sufficient to approximate the largest $\lambda$-contractive set $\mathcal{C}_{\max }^{\lambda}$ with a given precision $\epsilon$ using the sequence (44). Formally, this result is summarized in Thm. 9, Second, we showed in Thm. 12 how to compute a suitable $\lambda$ such that the associated maximal $\lambda$-contractive set is guaranteed to approximate the maximal controlled invariant set $\mathcal{C}_{\max }^{1}$ with a given accuracy. The statements in Thms. 9 and 12 were illustrated with an example. As one might expect, we found that the computed iteration bound $k$ and the provided choice for $\lambda$ are valid but conservative. Nevertheless, the procedure for a suitable choice of $\lambda$ guaranteeing (7) might be useful for practical computations of $\lambda$-contractive sets since the conservatism in $\lambda$ only slightly influences the termination of step-set based approximations of $\mathcal{C}_{\max }^{\lambda}$ (see the example in Sect. 5.2).

Theorems 9 and 12 both build on the contraction property summarized in Thm. 2 and the iteration bound introduced in Lem. 8 , The statements in Thm. 2 and Lem. 8 require the pair $(A, B)$ to be controllable (see Assum. 1) and this restriction is passed on to Thms. 9 and 12, Clearly, it would be desirable to extend all statements to systems that are "only" stabilizable. It was, however, shown that there exist stabilizable systems for which the statement in Thm. 2 does not apply (see the latter example in Sect. 5.3). Nevertheless, there is no fundamental argument against the extension of Thms. 9 and 12 to stabilizable systems. Consequently, future work has to address these non-trivial extensions in order to complete the theory. 


\section{Acknowledgment}

We thank the anonymous reviewers for their helpful comments and suggestions. Financial support by the German Research Foundation (DFG) through the grant SCHU 2094/1-1 is gratefully acknowledged.

\section{A. Additional proof}

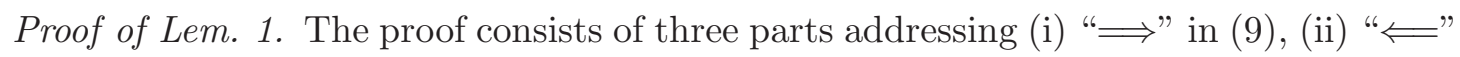
in (9), and (iii) relation (10). As a preparation, note that $\rho(\xi, \mathcal{C}) \leq \rho(\xi, \mathcal{D})$ for every $\xi \in \mathcal{S}$ due to $\mathcal{C} \subseteq \mathcal{D}$.

Part (i). Having $\mathcal{D} \subseteq \exp (\delta) \mathcal{C}$ implies $\rho(\xi, \mathcal{D}) \leq \rho(\xi, \exp (\delta) \mathcal{C})$ for every $\xi \in \mathcal{S}$. We further obtain $\rho(\xi, \exp (\delta) \mathcal{C})=\exp (\delta) \rho(\xi, \mathcal{C})$ for every $\xi \in \mathcal{S}$ by definition of $\rho(\cdot, \cdot)$. Thus

$$
d(\mathcal{C}, \mathcal{D})=\sup _{\xi \in \mathcal{S}} \ln \left(\frac{\rho(\xi, \mathcal{D})}{\rho(\xi, \mathcal{C})}\right) \leq \ln (\exp (\delta))=\delta
$$

Part (ii). Assume $d(\mathcal{C}, \mathcal{D}) \leq \delta$ but $\mathcal{D} \nsubseteq \exp (\delta) \mathcal{C}$. Then, there exists an $x \neq 0$ such that $x \in \mathcal{D}$ but $x \notin \exp (\delta) \mathcal{C}$. Define $\xi^{*}:=\frac{x}{\|x\|_{2}}$ and note $\xi^{*} \in \mathcal{S}$. Clearly, $\rho\left(\xi^{*}, \mathcal{D}\right)>\rho\left(\xi^{*}, \exp (\delta) \mathcal{C}\right)=\exp (\delta) \rho\left(\xi^{*}, \mathcal{C}\right)$. This, however, contradicts $d(\mathcal{C}, \mathcal{D}) \leq \delta$ since

$$
\ln \left(\frac{\rho\left(\xi^{*}, \mathcal{D}\right)}{\rho\left(\xi^{*}, \mathcal{C}\right)}\right)>\ln (\exp (\delta))=\delta
$$

Part (iii). Let $\mu^{*}:=\arg \min _{\mu} \ln (\mu)$ s.t. $\mathcal{D} \subseteq \mu \mathcal{C}$ and define $\delta^{*}:=\ln \left(\mu^{*}\right)$. Then, we have $\mathcal{D} \subseteq \exp \left(\delta^{*}\right) \mathcal{C}$ and consequently $d(\mathcal{C}, \mathcal{D}) \leq \delta^{*}$ according to part (i) of the proof. Now, assume $d(\mathcal{C}, \mathcal{D})=\delta<\delta^{*}$. Then, $\mathcal{D} \subseteq \exp (\delta) \mathcal{C}$ according to part (ii). This, however, contradicts $\mu^{*}$ being the optimizer of (10) since $\mu=\exp (\delta)<\mu^{*}$. Thus, $d(\mathcal{C}, \mathcal{D})=\delta^{*}$ in accordance with (10).

\section{References}

[1] F. Blanchini and S. Miani, Set-Theoretic Methods in Control. Birkhäuser, 2008.

[2] F. Blanchini, "Ultimate boundedness control for uncertain discrete-time systems via set-induced Lyapunov functions," IEEE Trans. Autom. Control, vol. 39, no. 2, pp. 428-433, 1994.

[3] D. P. Bertsekas, "Infinite-time reachability of state-space regions by using feedback control," IEEE Trans. Autom. Control, vol. 17, no. 5, pp. 604-613, 1972.

[4] M. Cwikel and P. O. Gutman, "Convergence of an algorithm to find maximal state constraint sets for discrete-time linear dynamical systems with bounded controls and states," IEEE Trans. Autom. Control, vol. 31, no. 5, pp. 457-459, 1986. 
[5] P. O. Gutman and M. Cwikel, "An algorithm to find maximal state constraint sets for discrete-time linear dynamical systems with bounded control and states," IEEE Trans. Autom. Control, vol. 32, no. 3, pp. 251-253, 1987.

[6] S. S. Keerthi and E. G. Gilbert, "Computation of minimum-time feedback control laws for discrete-time systems with state-control constraints," IEEE Trans. Autom. Control, vol. 32, no. 5, pp. 432-435, 1987.

[7] F. Blanchini and S. Miani, "Constrained stabilization of continuous-time linear systems," System and Control Letters, vol. 28, pp. 95-102, 1996.

[8] M. Fiacchini, T. Alamo, and E. F. Camacho, "On the computation of local invariant sets for nonlinear systems," in Proc. of 46 th Conference on Decision and Control, pp. 3989-3994, 2007.

[9] M. Schulze Darup and M. Mönnigmann, "On general relations between nullcontrollable and controlled invariant sets," in Proc. of 53th Conference on Decision and Control, pp. 6323-6328, 2014. 